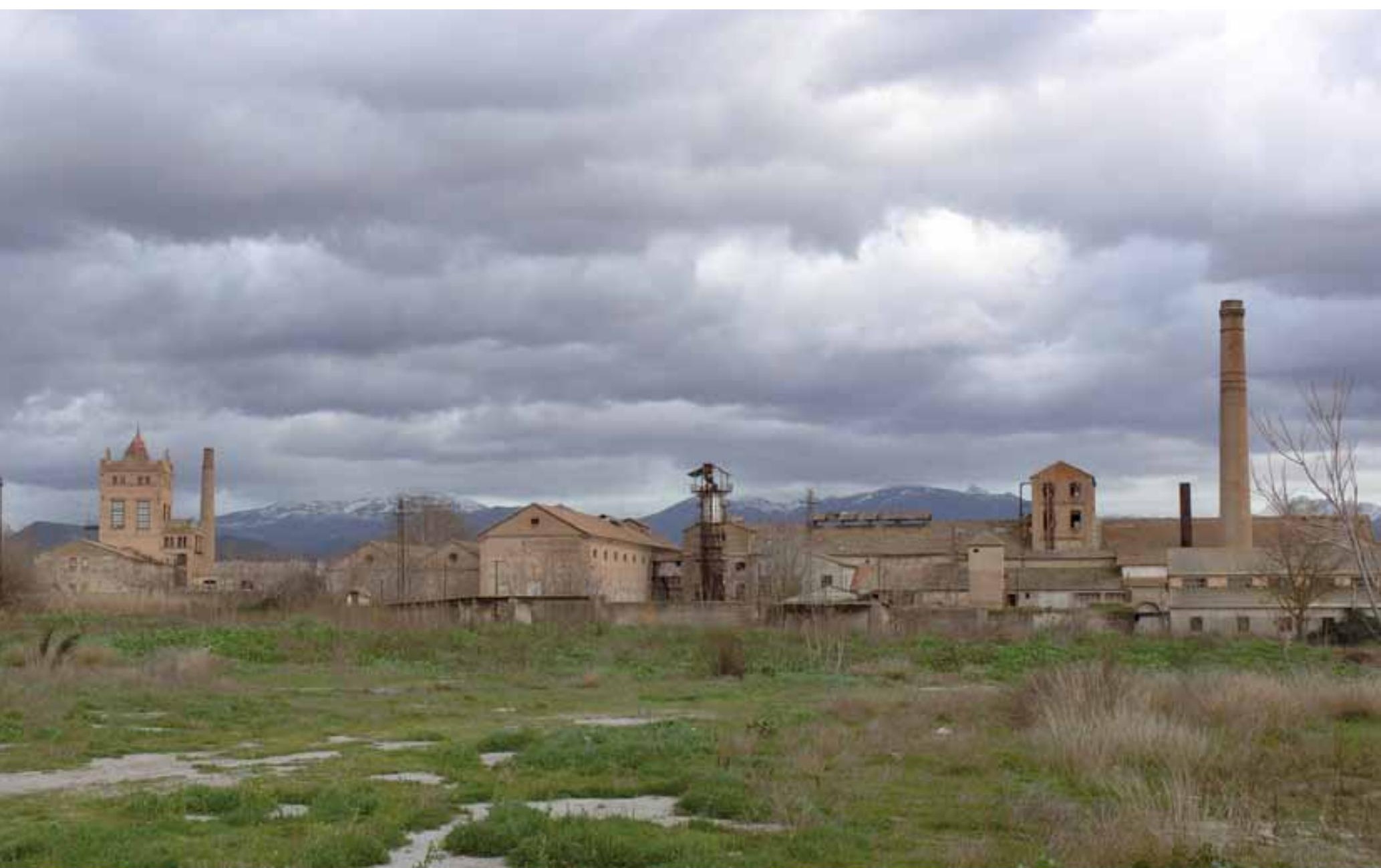

Conjunto fabril de San Isidro (Granada). Foto: José Castillo Ruiz 


\section{La vega de Granada. La construcción cultural de un territorio a través de la actividad agraria}

José Castillo Ruiz, Dpto. de Historia del Arte, U. de Granada
Desde el pionero y excepcional estudio de Carmen Ocaña (1974), la vega de Granada ha sido objeto de numerosos y variados estudios históricos, geográficos, paisajísticos, hidrológicos, urbanísticos, etc. que han aportado un conocimiento bastante amplio sobre las diferentes dimensiones que componen este espacio así como de su conformación a lo largo de la historia, y que han concluido con la caracterización de una comarca o un territorio bien definido desde el punto de vista geográfico aunque con las lógicas imprecisiones en cuanto a su formalización espacial. En este sentido, la vega del Genil constituye la unidad geográfico-comarcal más importante de toda la provincia de Granada por su población, superficie e importancia económica gracias a la fertilidad y riqueza de sus suelos así como a su relativa concentración fabril y, sobre todo, al desarrollo administrativo y mercantil de la ciudad de Granada. Esta comarca, que se corresponde con el fondo de la depresión del Genil o de Granada, tiene una extensión de $50 \mathrm{~km}$ de este a oeste, por 35 de norte a sur, y una superficie aproximada de unos $1.500 \mathrm{~km}^{2}$. Por lo que respecta al espacio de vega propiamente dicho, la superficie regada es, según datos de 2007, de 15.519 ha.

Paralelo (y en gran media alentado y aliado con él) a este reconocimiento científico ha ido cultivándose en la zona un sentimiento de aprecio y consideración por un espacio dotado de importantes valores culturales, naturales, sociales o económicos que exigen conocimiento y protección. Este sentimiento, expresado de forma muy diversa en cuanto a objetivos y personas (voluntariado medioambiental, defensa del tranvía, asociaciones de huertanos, agricultura ecológica, etc.), ha cristalizado en un movimiento ciudadano organizado en torno a la Plataforma Salvemos la Vega, la cual, después de una década de actividad, ha conseguido que para la mayoría de granadinos la vega de Granada sea un bien patrimonial con entidad propia y singularidad que merece consideración como tal. A él nos remitimos cuando hemos solicitado ante la Consejería de Cultura de la Junta de Andalucia su declaración como BIC, con la figura de Zona Patrimonial.

Todo este proceso descrito ha coincidido con el momento de mayor alteración y destrucción del espacio que históricamente ha conformado la vega como consecuencia del insostenible e irracional proceso de desarrollo urbanístico e industrial acaecido a partir de la década de los 80, sobre todo en las localidades más cercanas a la ciudad de Granada (Armilla, Las Gabias, Los Ogijares, Churriana, La Zubia, Atarfe, Maracena, etc.). Este proceso, al margen de la reducción significativa del espacio irrigado y cultivado históricamente, ha consolidado su condición periurbana al consagrar, a través de la conversión de estas localidades en pueblos dormitorio, su pertenencia al área metropolitana o aglomeración urbana de Granada. La aprobación del POTAUG (Plan de Ordenación Territorial de la Aglomeración Urbana de Granada) en 1999 legitimará esta condición, a pesar de la importancia de las medidas de ordenación y protección contenidas en él sobre el espacio ocupado por la vega, la cual pierde así su condición singular reconocida en otros instrumentos de ordenación especificos, aunque de nula aplicación, como el Plan Especial de Protección de la Vega de Granada aprobado en 1991. Ahora de nuevo, y como justificación y validación de un proyecto insensato como es la construcción del Parque del Milenio en terrenos de la vega sur, se está procediendo a elaborar por parte de la Consejeria de Vivienda y Ordenación del Territorio un nuevo Plan Especial de la Vega de Granada, el cual nace fallido ante el olvido del principal recurso y actividad de la vega: la agricultura y los agricultores.

Por tanto, la situación que actualmente presenta la vega de Granada es de incertidumbre en la que se combina la cautelosa esperanza de los movimientos de defensa ciudadana, la forzada y coyuntural atención de las administraciones públicas, la durmiente (aunque siempre expectante) posición de los especuladores y la crónica desazón (en algunos casos desesperanza e indignación) de los agricultores.

En esta situación, y colocándonos en el lugar de la esperanza y del futuro, queremos hacer un reconocimiento de los valores patrimoniales de la vega de Granada, que debe necesariamente partir de dos fundamentos: la identificación de la actividad agraria históricamente desarrollada como el elemento generador de la dimensión patrimonial de la misma y su condición de 

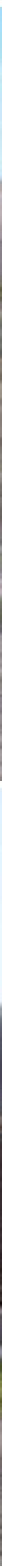

espacio singular y con entidad propia, lo que exige trasladar la histórica contemplación de la vega desde "la Torre de la Vela" para hacerlo desde "el Cortijo del Pino", donde no necesitamos escuchar los tañidos de la campana de la Vela -tantos siglos utilizada por los agricultores para la organización del regadio-, pues hace tiempo que miramos hacia otro lado, hacia nosotros mismos, hacia el futuro.

\section{LA VEGA DE GRANADA COMO PATRIMONIO CULTURAL. VALORES Y TIPOS DE BIENES}

La vega de Granada adquiere singularidad, valor y reconocimiento social fundamentalmente por su condición agraria, más aún, por el hecho de ser un espacio cultivado a lo largo de la historia. Por lo tanto la caracterización patrimonial que debe hacerse de ella, y que aqui sólo dejamos apuntada, es la de identificar todos aquellos bienes materiales e inmateriales conformados por la

actividad agraria a lo largo de la historia, comenzando por el elemento que la estructura, posibilita y delimita como tal espacio agrario $y$, sobre todo, como ámbito patrimonial: el sistema hidráulico. A partir de aqui deberán ir superponiéndose a la tela de araña tejida con respeto y memoria histórica por la red de acequias, el resto de manifestaciones patrimoniales, entre otras: la arquitectura agrícola y los asentamientos poblacionales, las vías de comunicación, los cultivos, el paisaje y, finalmente, la ejemplarizante y múltiple presencia de Federico Garcia Lorca, cuya partida a la capital, pero regreso permanente a su familiar Asquerosa (Valderrubio), simboliza muy bien qué es y qué queremos que siga siendo la vega.

\section{El sistema hidráulico}

Con independencia de los antecedentes romanos existentes, el sistema de riego que da lugar al nacimiento de la vega de Granada se construye en la época medieval islámica. Si bien la noticia 

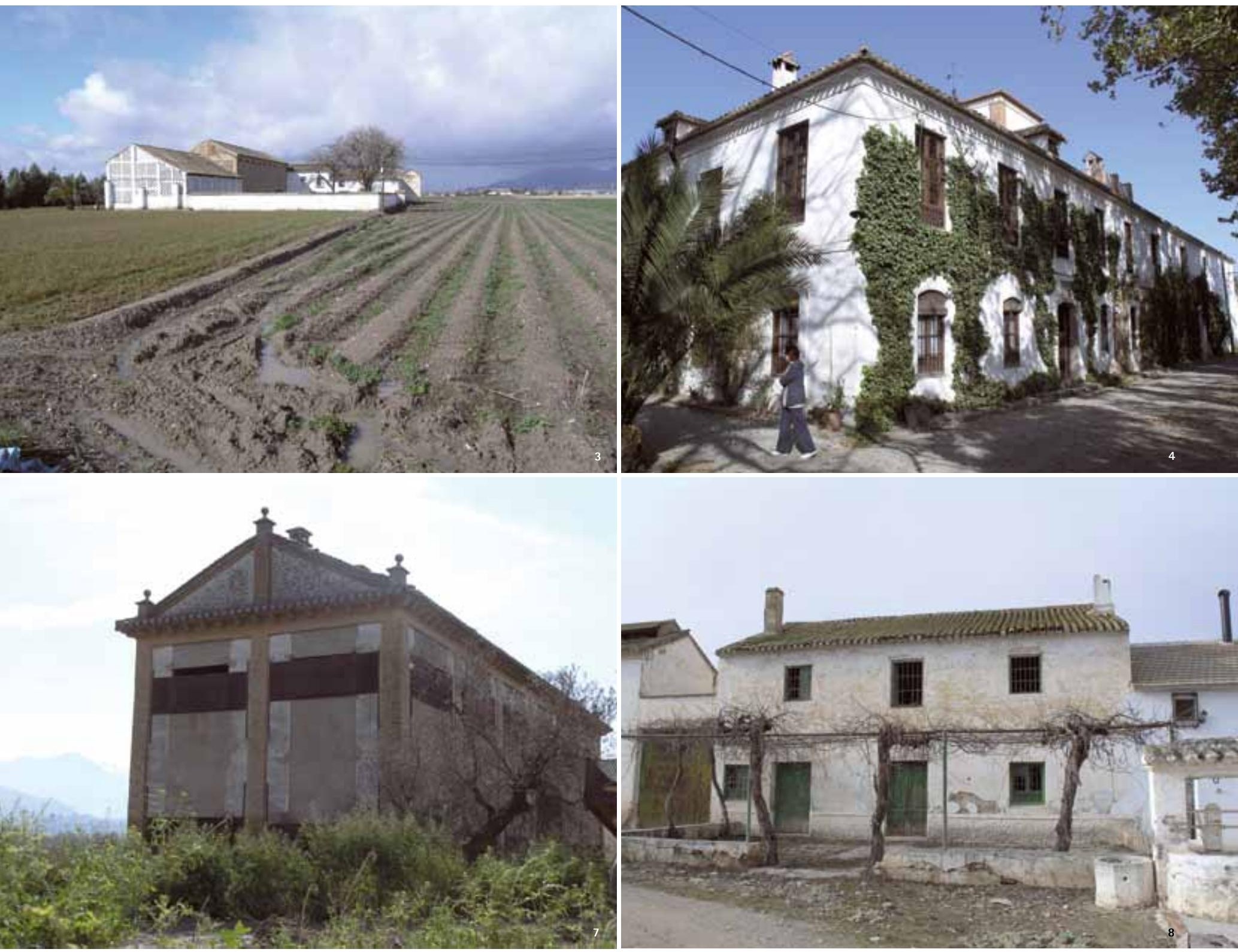

documental más antigua que disponemos del riego en la vega es el repartimiento de las aguas del Genil de 1219 (Repartimiento de Abd Allah), en época almohade, el trazado de sus elementos más importantes estaba ya configurado desde la época emiral o califal, alcanzando pleno desarrollo en época ziri y nazarí. Esto significa, y queremos resaltarlo especialmente, que la vega de Granada existía con anterioridad a la propia fundación de la ciudad de Granada, cuyo origen debemos situar en el siglo XI (MALPICA CUELLO, 1997: 231).

Lo extraordinario de este sistema hidráulico, que es el que ha permitido conformar la organización productiva, social y urbana de la vega de Granada, es su pervivencia, y además con un alto grado de autenticidad, en el presente, manifestada, por un lado, en la conservación del trazado originario del sistema de riego -lo cual debe equipararse en relevancia patrimonial al mantenimiento del entramado urbano del Albaicin musulmán- y, por otro lado, en su continuidad funcional, persistiendo las formas de organización y
1. Relación de la vega con la ciudad de Granada.

2. Vega de Monachil.

3. Cortijo de la Mona. Pago de Naujar (Granada).

4. Cortijo del Pino (Churriana de la Vega, Granada).

5. Canal de Las Madres de Rao (Santa Fe).

6. Tránsito por la vega de Pinos Puente.

7. Secadero del cortijo de Gallardo (Granada).

8. Cortijo de Daimuz Alto. Propiedad de la familia Lorca de 1903 a 1908

(Valderrubio, Pinos Puente).

Fotos: José Castillo Ruiz 


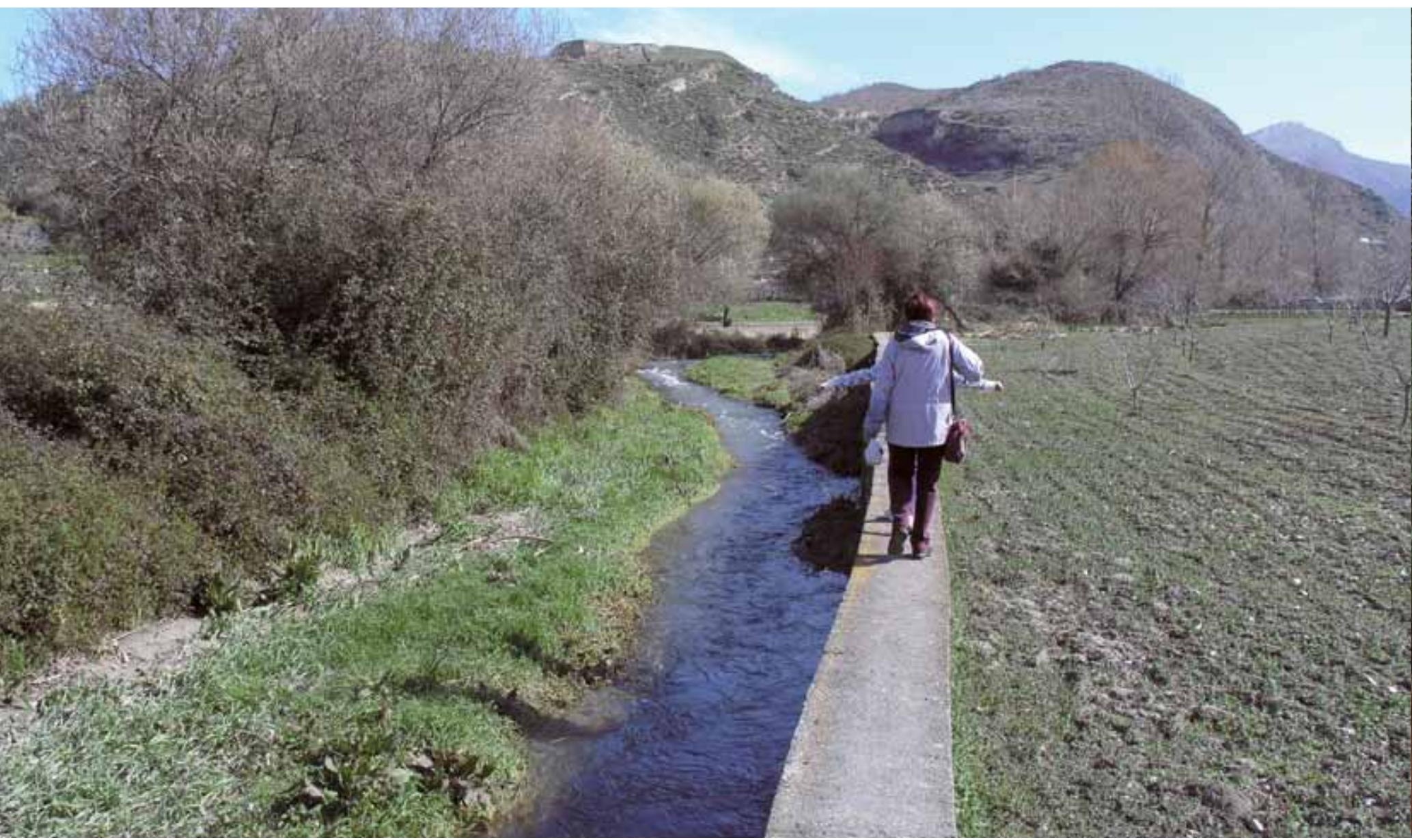

Captación de agua de la Acequia Gorda de la Zubia. Río Monachil (Monachil). Foto: José Castillo Ruiz

gestión del riego: la distribución del agua por pagos, la organización autónoma de cada acequia o incluso la organización del riego en la parcela.

De los muchos y diversos elementos que conforman el sistema de riego, los más importantes y reconocibles en la actualidad son las acequias, la mayoria de ellas existentes desde época islámica, como, por citar algunas de las principales, las acequias Gorda, Arabuleila y Tarramonta (que captan el agua del río Genil aguas arriba de la ciudad de Granada), las acequias Gorda de La Zubia, Genital y la Estrella (procedentes del río Monachil), las acequias del Dílar, Real de los Ogijares y de Alhendín (que captan en el río Dílar) o las acequias de En Medio, La Cruz y Alitaje (del río CubiIlas). Respecto al resto de elementos del sistema hidráulico destacamos los azudes (como el azud sobre el río Cubillas en Atarfe o las denominadas Medias Lunas de Pinos Puente), los molinos, fundamentalmente harineros, o los importantes, histórica y ambientalmente, canales que conducen el agua de los manantiales hasta las acequias como, por ejemplo, los de San Juan, San José o el de las Madres del Rao.

\section{Arquitectura agrícola y asentamientos poblacionales}

Al margen de la importancia que debemos otorgar a la arquitectura popular y estructura urbana de las localidades que integran la vega de Granada, las cuales apenas disponen de reconocimiento y medidas de protección, podemos identificar dos unidades o tipos de explotaciones agrarias claramente reconocibles: Ios cortijos y las huertas (TORICES ABARCA; ZURITA POVEDANO, 2003). Si bien comparten muchas características comunes -se trata de explotaciones polivalentes de entidad funcional y constructiva mediana con importante presencia del núcleo residencial y con una organización por lo general nuclear, aunque también en torno a patios poco articulados que aglutinan las dependencias para el ganado, almacenamiento 0 , en algunos casos, para la transformación-, la diferencia radica fundamentalmente en la relevancia del módulo residencial, mucho mayor en el caso de las huertas -cuya continuidad histórica respecto a las almunias o casas de recreo árabe les otorga un valor añadido (TRILLO SAN JOSÉ, 2004)--, donde la vivienda del propietario adquiere un importante desarrollo con la inclusión de elementos formales y compositivos procedentes de la arquitectura urbana, al utilizarse en muchos casos como segunda residencia.

Algunos de los ejemplos más sobresalientes de huertas son: la huerta del Tamarit, huerta del Marqués, huerta de los Perejileros y huerta Corazón de Jesús en Granada; la huerta en Cúllar Vega, la huerta del Marqués en Otura; la huerta de Nuestra Señora del Pilar en Vegas del Genil y la huerta Grande y Casa Muharra en la Zubia. Por lo que respecta a los cortijos, destacamos los siguientes: cortijo del Pino en Churriana de la Vega; cortijo de 


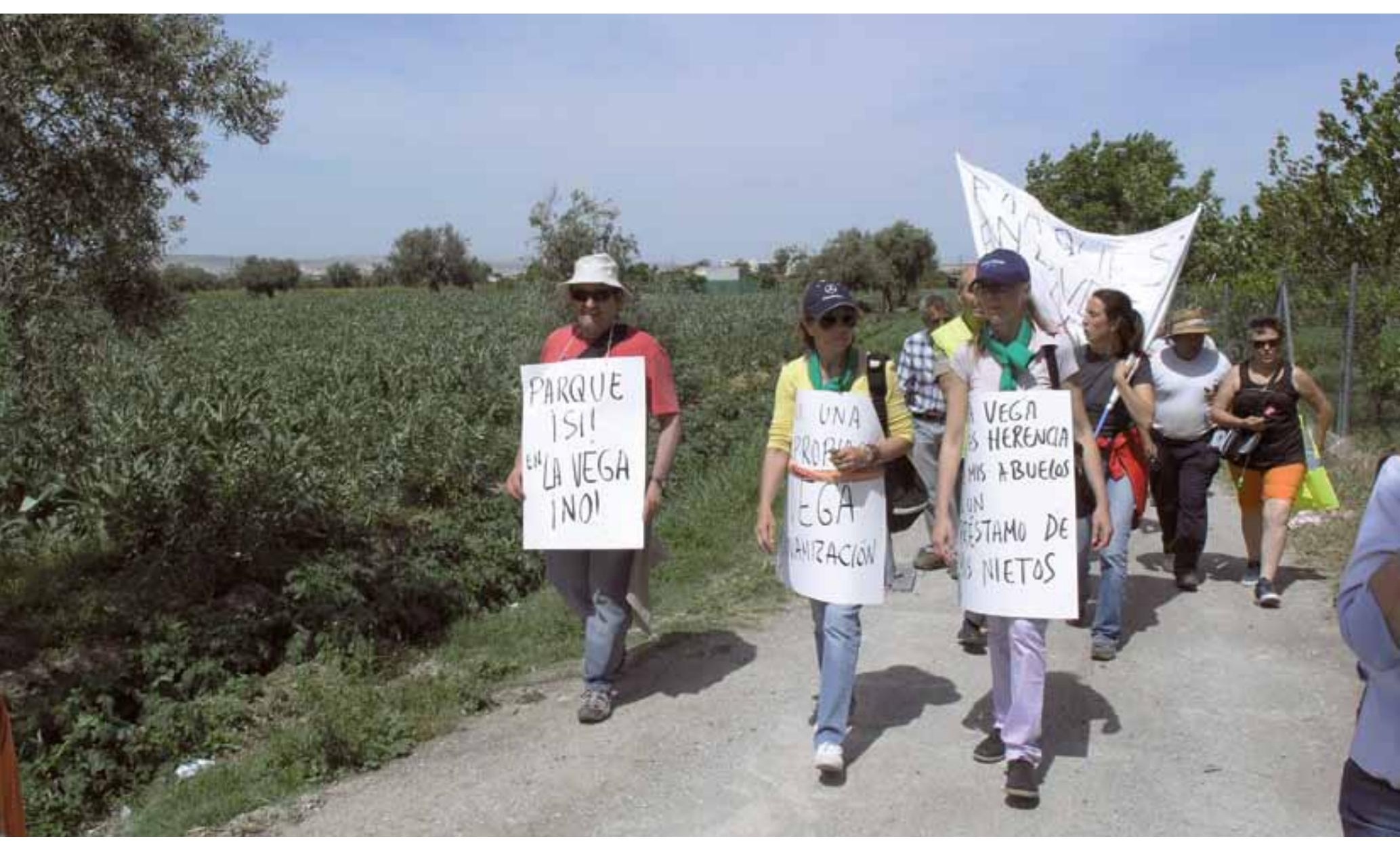

Marcha reivindicativa Salvemos la Vega en mayo de 2009. Foto: José Castillo Ruiz

la Viña en Cúllar Vega; cortijo de la Cartuja, cortijo del Rector, cortijo de Santa Maria de la Vega, cortijo de Alarcón y cortijo del Cobertizo en Granada; caseria de Santa Ana, cortijo del Alitaje, cortijo de Daimuz Alto y cortijo de la Marquesa en Pinos Puente; cortijo de Santa Teresa en Santa Fe y caserio de San Ignacio en Vegas del Genil.

\section{Tránsitos y vías de comunicación}

El otro gran elemento estructurador de la vega de Granada lo constituyen las vías de comunicación y tránsito, ya que permiten entender el grado de interconexión entre los diferentes núcleos de población, la relación entre los lugares de residencia y los espacios de producción agraria o, finalmente, la convivencia entre la explotación agraria y ganadera.

Las vias de comunicación o espacios de tránsito que podemos identificar en la vega de Granada son las carreteras de comunicación entre los diferentes pueblos y con Granada, los caminos agrícolas de diferente nivel, las líneas del tranvía eléctrico (en menor medida las líneas de ferrocarril) y las cañadas o vías pecuarias. Si bien lo que más caracteriza patrimonialmente a estas vías históricas es su enorme alteración, cuando no destrucción, consideramos que todavía existen vestigios materiales importantes que sería posible recuperar $y$, por tanto, preservar. Por ejemplo, la red de caminos agrarios secundarios, muchos de ellos todavía sin asfaltar, que permiten el acceso al compartimentado parcelario desde los diferentes núcleos de residencia (pueblos y cortijos), claro reflejo de la histórica división y fragmentación de la propiedad en la vega de Granada.

\section{Cultivos}

Desde la perspectiva del patrimonio agrario, que es como debe abordarse la valoración patrimonial de cualquier espacio agrícola, las especies cultivadas adquieren un protagonismo esencial, ya que si bien son muchos los factores que determinan la implantación de uno u otro cultivo, son éstos, con sus particulares exigencias de plantación, riego, abonado, recolección, almacenaje, comercialización, transporte y, en su caso, transformación, los que van a configurar los vestigios materiales e inmateriales del patrimonio agrario, en este caso de la vega de Granada, otorgando al proceso económico o productivo agrícola entidad y reconocimiento patrimonial.

Desde este punto de vista, y debido al policultivo existente en la vega de Granada, así como a su variación a lo largo de la historia, resulta difícil realizar una valoración del patrimonio generado por las especies cultivadas en ella a lo largo de la historia, lo cual requerirá de estudios futuros. No obstante, teniendo en cuenta el grado de conocimiento existente en la actualidad, podemos concluir que existen, o más bien han existido, dos cultivos que han 
generado un importante y variado patrimonio, cuya persistencia en la actualidad identifica de forma muy precisa la riqueza e identidad patrimonial de la misma. Nos referimos a la remolacha y al tabaco.

En el caso de la remolacha, cuya expansión se produce especialmente en la primera mitad del siglo $X X$, contribuyendo su cultivo y explotación al mayor impulso modernizador alcanzado por la provincia en todo este siglo, los vestigios patrimoniales más relevantes son los de carácter industrial relacionados con la producción de azúcar. Destacamos especialmente el conjunto fabril de San Isidro, situado en el término municipal de Granada, en el cual se integra el ingenio de San Juan (1882), la alcoholera de San Pedro (1886), la azucarera de San Isidro (1901) y la alcoholera de San Isidro (1908); la azucarera del Genil S.A, La Purisima Concepción, también en Granada (1905); la azucarera de la Vega, en Atarfe (1904-05), o la azucarera de San Pascual, en Pinos Puente (1910).

Junto a la remolacha, que es el cultivo que podemos considerar mítico en la vega, el otro cultivo que ha generado un mayor patrimonio es el tabaco (predominante en la vega desde la década de los 40 hasta la de los 70, que es cuando comienza su decadencia), sobre todo a través de los omnipresentes secaderos, que los podemos considerar como el elemento patrimonial identificador o símbolo de la vega de Granada. Las características de los secaderos son su elevado número existente, su dispar calidad, destacando por sus cualidades arquitectónicas y formales los realizados en ladrillo, y su dispersión, aspectos todos ellos que reflejan un hecho consustancial a la historia productiva de la vega: la fragmentación de la propiedad. Frente a las indicaciones de las autoridades de construir grandes secadores comunales, los agricultores optaron por construir los que necesitaban para su explotación, los cuales podian hacerse junto a la propiedad, más habitual en el caso de que existiera un cortijo, a cuyas construcciones se suelen adosar (los cortijos más importantes señalados anteriormente disponen prácticamente todos de secaderos, caso, por ejemplo, del cortijo del Alitaje en Pinos Puente, el de Santa María de la Vega en Granada o el de Calderón en la Zubia), en los núcleos urbanos (algunos de los secaderos urbanos más interesantes los encontramos en Pinos Puente, Fuente Vaqueros, Belicena o en la barriada de Bobadilla en Granada) o, así mismo, agrupados con otros formando conjuntos de secaderos como los del camino del Jau a Pedro Ruiz. Este hecho es el que explica que existan secaderos de tan diferente manufactura o calidad, ya que reflejan la capacidad económica del propietario.

\section{Valores paisajísticos}

La vega de Granada es ante todo un espacio unitario, tanto en su conformación física e histórica como en su apropiación por la ciudadania, de ahi que uno de los valores más importantes de la misma sea el paisajístico, que se corresponde con estos criterios de unidad y percepción.
Como señalan los autores del informe Ordenación y Protección de la Vega de Granada, de julio de 19981, la vega de Granada se ofrece al observador como una gran cuenca visual autocontenida que permite un alto nivel de intervisualización desde su interior a los bordes y viceversa debido a su conformación como cuenca sedimentaria rodeada de glacies y montañas y que posibilita la creación de numerosas y variadas perspectivas visuales. Destacamos al respecto, al margen de los excepcionales miradores existentes en la ciudad de Granada, especialmente en la Alhambra y el Albaicin, los del cerro de la Ermita de los tres Juanes en Sierra Elvira (Atarfe) y el de Montevives, en Gabia la Grande. Esta configuración descrita es la que produce, según los autores comentados, la otra caracteristica paisajística de la vega: el intenso contraste entre ésta y su entorno envolvente.

\section{Federico García Lorca: símbolo del valor y reconocimiento de la vega de Granada}

Federico García Lorca, a través del reconocimiento sobre el territorio de la vega de su presencia vital y creativa, se está convirtiendo en el símbolo de la recuperación de ésta como espacio patrimonial. Si bien su identificación con la ciudad de Granada resulta legitima y necesaria, apenas quedan vestigios materiales de su presencia en la misma, mientras que, por el contrario, son numerosos los que aún se mantienen diseminados por la vega, tanto en Fuente Vaqueros, donde nació el 5 de junio de 1898, como, sobre todo, en el cercano pueblo de Valderrubio (Ilamado Asquerosa en el momento en el que el vivía), donde se trasladó con su familia en 1905 y en el que permanecería hasta su marcha a Granada en 1909, lo que no impidió que siguiera volviendo de forma temporal hasta que compraran en 1925 la Huerta de San Vicente como residencia de verano (en ese momento en plena huerta de Granada). En este pueblo nos encontramos, además de la casa familiar (convertida actualmente en museo), la vivienda prácticamente intacta de Frasquita Alba, la familia vecina que inspiró el drama de la casa de Bernarda Alba, el cortijo de Daimuz, donde su padre consiguió, cómo no, a través del cultivo de la remolacha, gran parte de los recursos que le permitieron posteriormente trasladarse a la ciudad de Granada, la fuente de la Carrura o de la Teja, donde Lorca aprendió a interpretar el sentir popular, o multitud de lugares más donde persiste la memoria de muchos de los personajes (sus herederos ya) que habitan sus obras.

\section{Notas}

'La redacción de este documento, realizado como soporte documental para la elaboración del Plan de Ordenación del Territorio de la Aglomeración Urbana de Granada (POTAUG), ha sido llevada a cabo bajo la dirección técnica de Gloria Vega González y ha contado con la colaboración de los miembros de la Oficina Técnica de la Aglomeración Urbana de Granada. 